\title{
Verification of Strain Transfer Characteristics of Distributed Surface-attached FBGs on Aircraft Wings
}

\author{
Zhen Ma and Xiyuan Chen*
}

Key Laboratory of Micro-Inertial Instrument and Advanced Navigation Technology, Ministry of Education, School of Instrument Science and Engineering, Southeast University, 210096 Nanjing, China

\begin{abstract}
In order to provide higher accuracy for transfer alignment on aircraft, a surface-attached FBGs is designed for distributed deformation measurement of aircraft wing. To verify the measurement accuracy of the surface-attached FBGs, a strain calibration method of the surface-attached FBGs applied with the wing strain measurement is proposed. Two Aluminium Alloy Test Plates(AATP) with different sizes were designed as the matrix material for tensile test. The test results show that AATP-A has better test effect. Through the two sets of tensile tests, the change of wavelength measured on the surface-attached FBGs is in good agreement with the change of strain value of strain gauge. The experimental results prove that the method based on surface-attached FBGs is feasible for the strain measurement of aircraft wing. The method is simple and reliable, which lays a foundation for the follow-up study of the full-field deformation measurement of aircraft wing.
\end{abstract}

\section{Introduction}

Structural health monitoring has always been an important research area in the field of aerospace. The deformation extent of aircraft wing directly affects the flight safety of aircraft. Airborne distributed measurement is an effective means for wing deformation monitoring. Fiber Bragg grating (FBG) has many advantages in distributed measurement of aircraft wing, among which the strain calibration technology of FBGs is a hot topic. For FBG strain calibration, researchers have done a lot of research work and achieved important results. ${ }^{[1-2]}$ Tavares et al. ${ }^{[3]}$ designed a method to calibrate tube packaged FBGS in a wide range by four-point bending test, and established the finite element model of plastic bending deformation of four-point bending test beam. Rothsch et al. [4] established a strain calibration device for FBG strain sensor according to the German VDI/VDE 2660 guide. The measuring accuracy of the device is high, but its structure is complex. Habel et al. ${ }^{[5]}$ of Germany developed a calibration test device for FBGs. The unbiased digital image correlation (DIC) and electronic speckle interferometer (ESPI) are used for calibration measurement. The KALFOS (calibration of fiber optic sensors) facility designed by researchers consists of a load bearing facility and a temperature chamber. The experimental results show that the KALFOS method can analyze their strain transfer mechanism, and can easily observe the relative motion between sensors and samples which helps to validate the definition and quantitative description of component requirements in new or existing standards. This method has high accuracy. Lazarev et al. ${ }^{[6]}$ designed a non-destructive calibration device for encapsulated FBG strain sensor using mechanical nano-motion sensor. Through the analysis of experimental data, the performance of FBG strain sensor is studied, the calibration curve of FBG strain sensor is given, which verifies the feasibility of this method. From these we can see that researchers have done a lot of research on calibration of tube-packaged FBGs, and the calibration technology is relatively mature on tube-packaged FBGs. However, there are few literatures about the calibration method of surface-attached FBGs, and there is no mature technical method.

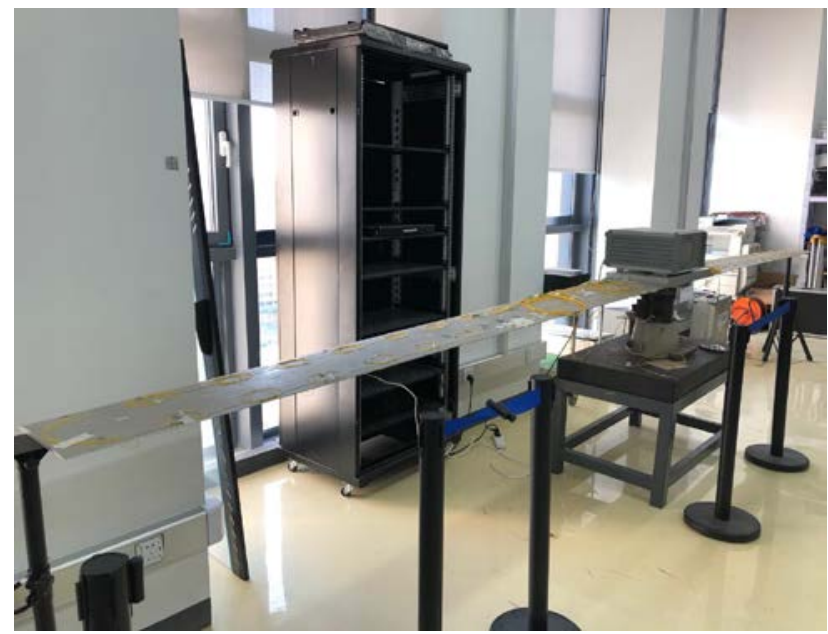

Fig.1. Aircraft wing model

Corresponding author: chxiyuan@seu.edu.cn 
As shown in Fig.1, in order to study the strain transfer effect of surface-attached FBG in wing deformation measurement, we simulated the real wing structure design and designed the wing model. Several distributed fiber Bragg gratings are attached to the aircraft wing. The strain transfer characteristics of surface-attached FBGs are studied experimentally. Two specimens of different sizes were designed to verify the calibration method designed in this paper. The feasibility of surface-attached FBGs strain measurement is emphatically analyzed through comparative experiments.

\section{Test equipment}

As shown in Fig.2, in order to ensure the accuracy of fiber wavelength measurement, KL-530 optical fiber fusion splicer is used in the selection of test equipment to ensure the accuracy of fiber grating. The interrogation technique adopts SM130 interrogation technique made by U.S.A.. Electro-mechanical Universal Testing Machines (EUTM) is produced by Shenzhen Sansi Longitudinal and Horizontal Technology Co., Ltd. with a measuring range of $5000 \mathrm{~N}$. DH3818Y static strain gauge is used to read the strain value of strain gauge in the experiment at a frequency of $1 \mathrm{~Hz}$. Besides these, two aluminium alloy test plates (AATP) of different sizes are designed in this paper. As shown in Fig.3, AATP-A is $260 \mathrm{~mm} \times 60 \mathrm{~mm} \times 3 \mathrm{~mm}$, and AATP-B is $200 \mathrm{~mm} \times 35$ $\mathrm{mm} \times 8 \mathrm{~mm}$. In order to transfer strain effectively, a fast-drying 3 ton epoxy adhesive was used to fix FBG and strain gauge. In the experiment, computer-1 was used to record the wavelength value, computer-2 was used to record the strain value, and computer- 3 was used to record the tension change. Fiber Bragg Grating is sensitive to temperature change, so we conduct experiments under constant temperature environment. Under constant temperature, the change of wavelength value is proportional to the change of strain value.

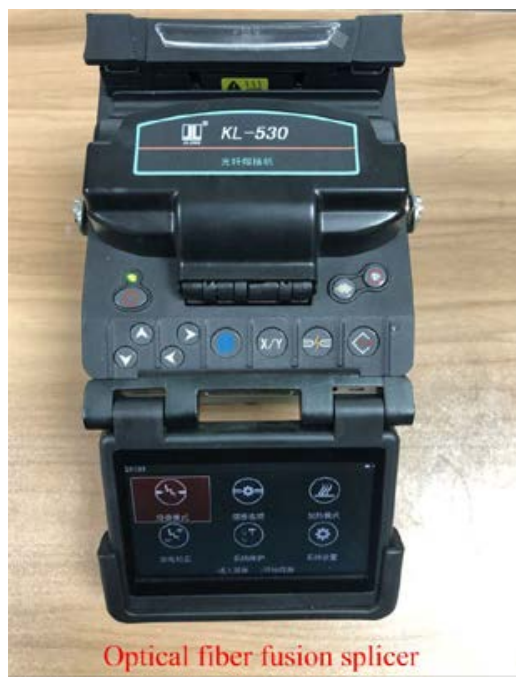

(a)

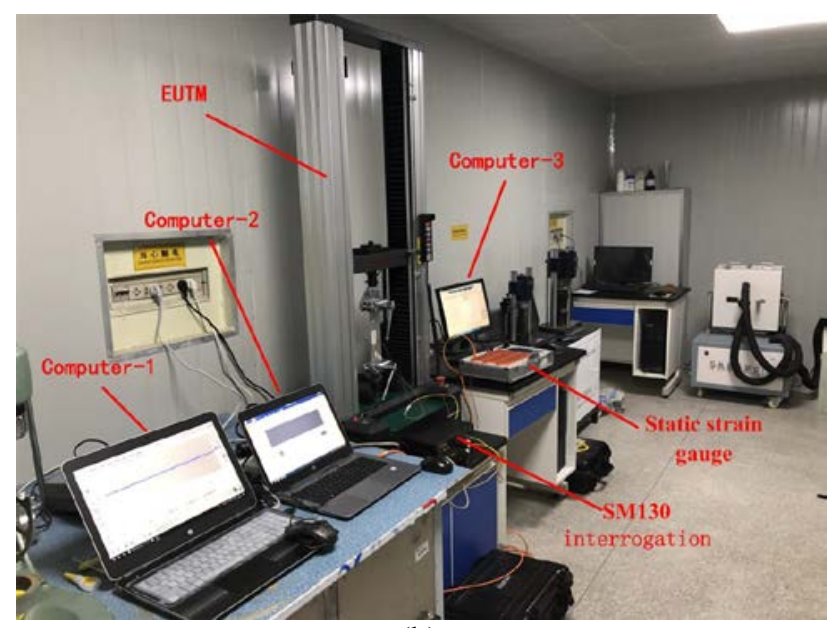

(b)

Fig.2. Test equipment

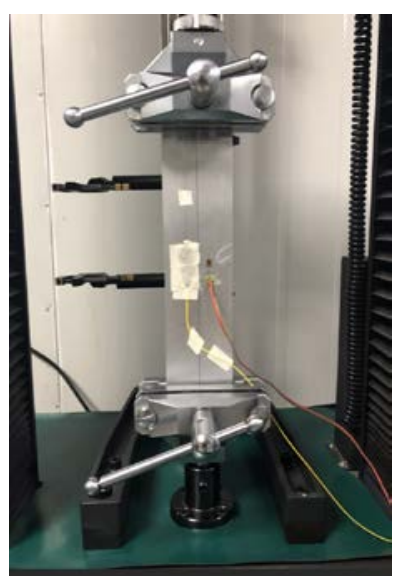

(a) AATP-A

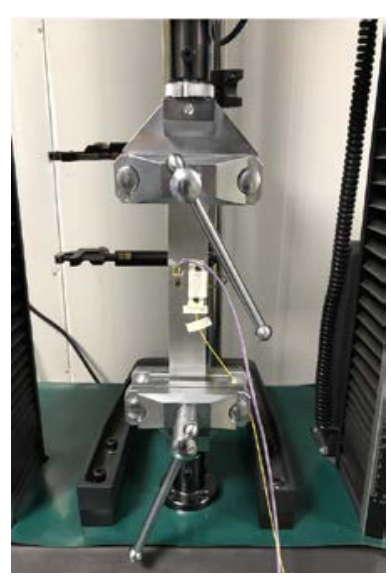

(b) AATP-B
Fig.3. Different sizes of AATP

\section{Experimental methods and data analysis}

\subsection{Analysis of Strain Transfer Characteristics of Different AATP}

In order to test whether the strain transfer effect of FBGs on different size specimens is consistent under the same force, we first carried out the tensile test of AATP-A. The test data show that the data obtained by FBGs on AATP-A specimen have good linearity. Then, we carried out tensile test on AATP-B specimen, the test data showed that the data obtained by FBGs on AATP-B specimen changed abnormally, and the test results were not as good as those on AATP-A specimen.

In order to study whether the abnormal changes of AATP-B data are caused by human factors, we conducted two experiments. As shown in Fig.4, in the first group of experiments, by observing the trend change of curve-1, we can find that when $\mathrm{F}>1 \mathrm{kN}$, the trend of curve-1 changes abnormally and the curve-1 will not continue to increase with the increase of force, it will jitter and slide. Meanwhile, the initial value of curve- 1 is not 0 , which means that AATP-B may have residual 
force at the initial time. On the premise of avoiding the possible interference factors in the first experiment, we conducted the second group of experiments on AATP-B. The trend of curve- 2 shows that the reverse force appears in the first five seconds of tensile test. When $\mathrm{F}>1.3 \mathrm{kN}$, the trend of curve- 2 shows obvious non-linear change, and the value of force detected on computer- 3 shows a similar decline of jitter.

Through the analysis of these two sets of test data, it can be concluded that the main factors affecting the abnormal change of the curve are the residual force caused by inadequate clamping force, slip and unreasonable clamping. Therefore, in order to improve the measurement accuracy and avoid the interference factors in the first two groups of tests, we need to pre-load specimen before the test to remove the residual stress. At the same time, the results of data analysis of two groups of AATP-B test show that AATP-A test measurement effect is better than AATP-B, so we choose AATP-A as the test material to verify the strain transfer effect of surface-attached FBGs.

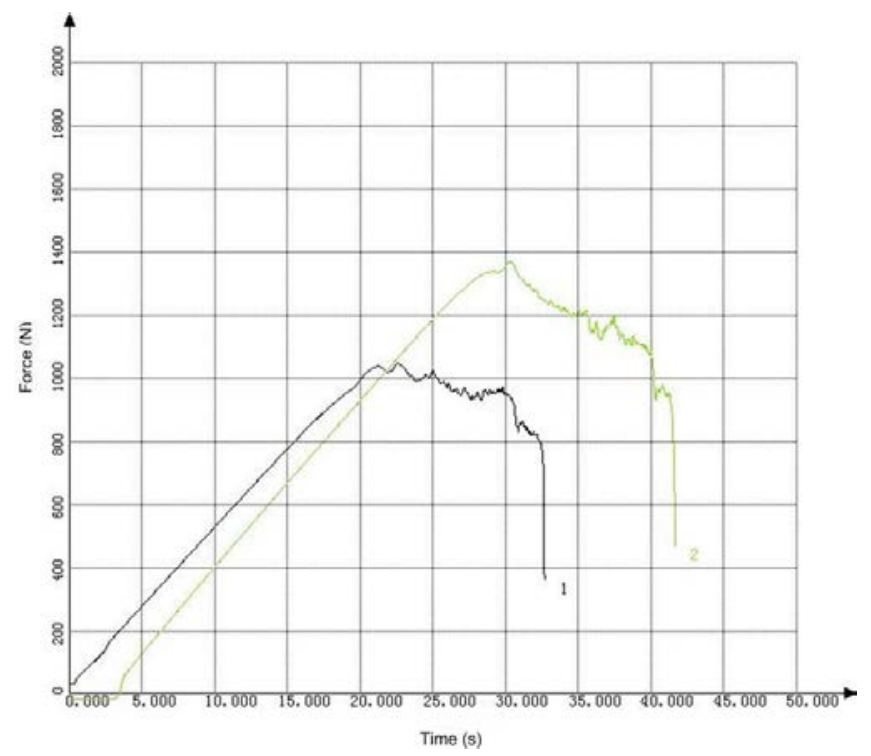

Fig.4. Curve of tensile test

\subsection{Analysis of Strain Transfer Characteristics}

Before strain transfer characteristic analysis test, in order to detect the maximum range on AATP-A and determine the optimal test method for AATP-A, this paper applied equal gradient increasing force on AATP-A until the force on AATP-A changed abnormally. Firstly, the force is applied to $300 \mathrm{~N}$ at a speed of $50 \mathrm{~N} / \mathrm{s}$, then the pull force is maintained for $60 \mathrm{~s}$, and then the previous operation is repeated. As shown in Fig.5, when the force is greater than $1.5 \mathrm{kN}$, the value of the force measured on EUTM changes abnormally. For this reason, the maximum range of the force applied in this experiment is set to $1.5 \mathrm{kN}$.

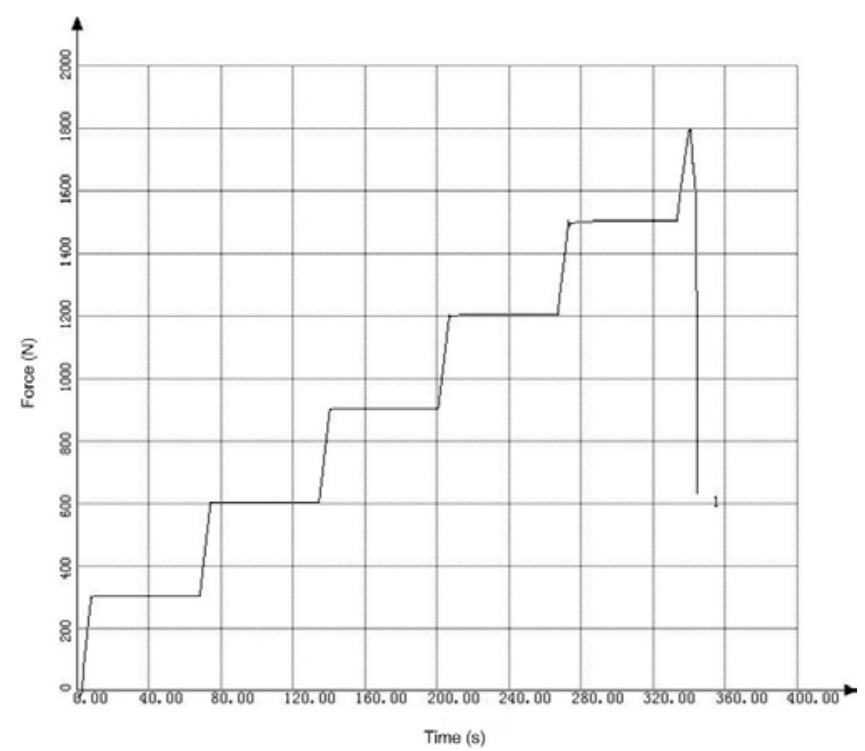

Fig.5. Maximum Range Test

In order to validate the strain transfer characteristics of surface-attached FBGs effectively, the strain transfer effect of surface-attached FBGs is validated by applying uniform increasing force. When applying tension to AATP-A by EUTM, it increases from 0 to $1.5 \mathrm{kN}$ at a speed of $50 \mathrm{~N} / \mathrm{s}$, then maintains for 60 seconds, and finally unloads AATP-A. The experiment was repeated twice, and then the data were observed and analyzed. The variation of wavelength values in two groups of tension tests is shown in Fig. 6.

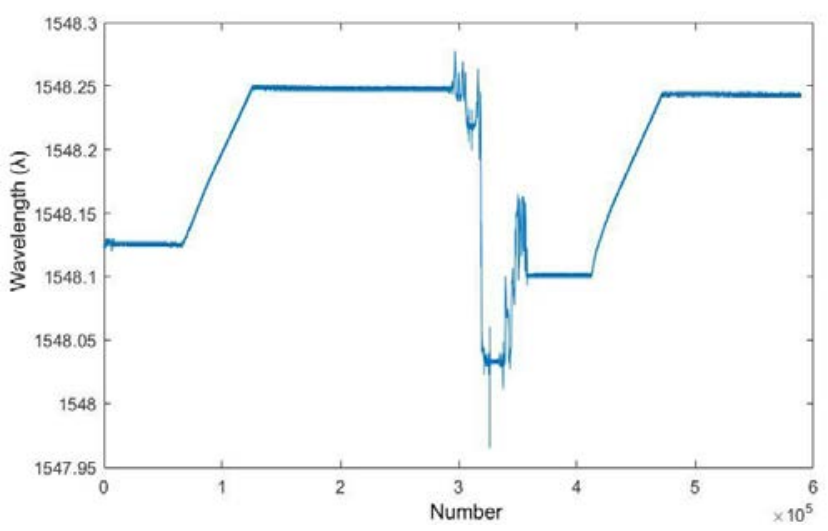

Fig.6. Wavelength value of tension test

From Fig.6, we can see that the working conditions of the two groups are the same, the initial values of the wavelength values in the two groups are different. The initial wavelength of the second group is less than the initial wavelength of the second group. When the tension is applied to the maximum value, the maximum wavelength of the two groups is basically the same. 


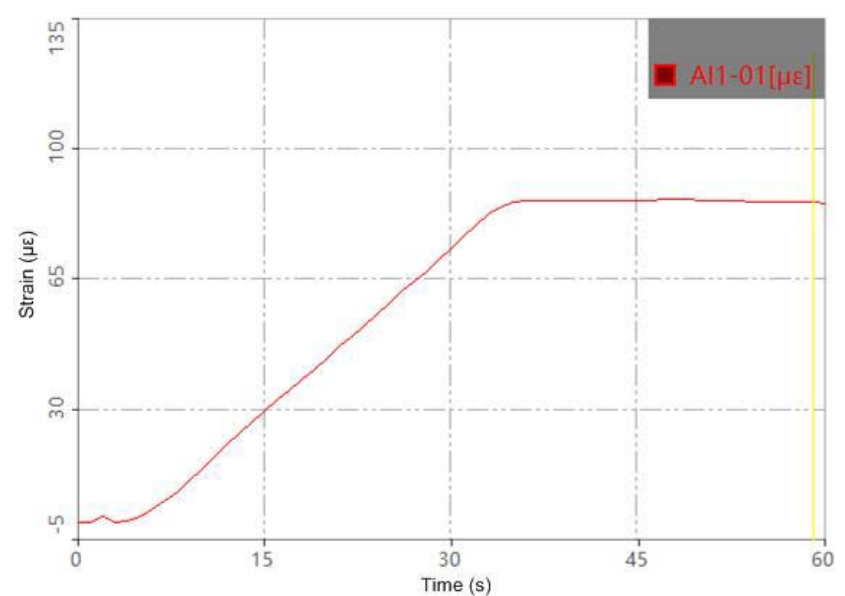

Fig.7. Strain value of the first tension test

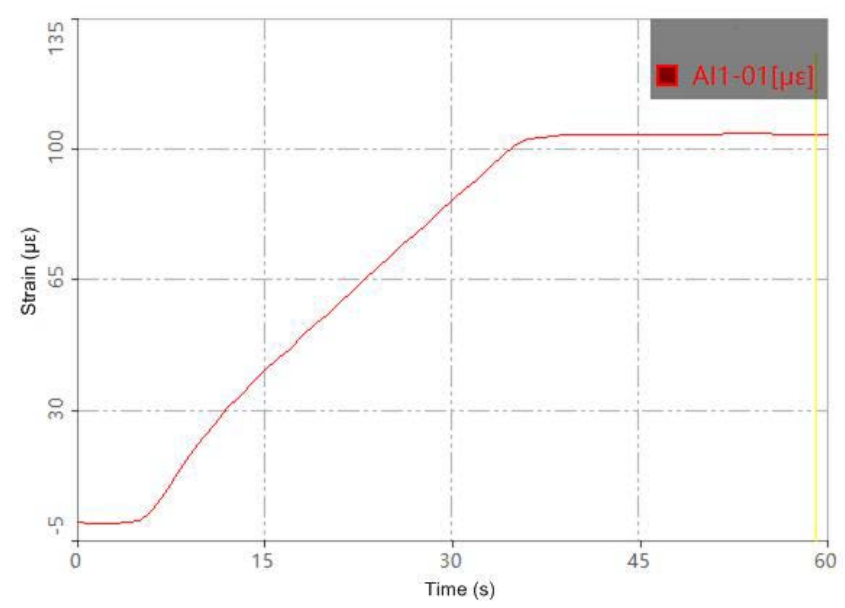

Fig.8. Strain value of the second tension test

In the meantime, we use strain gauges to obtain two sets of strain values. Fig.7 and Fig.8 show the changes of strain values measured by static strain gauge during two sets of tensile tests on AATP-A. Through two groups of experiments, we can find that the wavelength measured by the surface-attached FBGs and the strain value measured by the static strain gauge have good consistency. From Fig.7, we can find that the strain value fluctuates in a small range at the beginning. In the first group of experiments in Fig.6, the wavelength value fluctuates in a small range too. In this experiment, the static strain gauge itself has a high measurement accuracy. The experimental comparison proves that the change of wavelength has a good match with the strain value, that is to say, the surface-attached FBGs has a good strain transfer characteristics.

\section{Conclusions}

In order to verify the strain transfer characteristics of surface-attached FBGs, a strain transfer test verification method of surface-attached FBGs is designed. The test result of AATP-A is better than that of AATP-B. Then, AATP-A is used for tensile test to compare the changes of the measured wavelength and strain values. The test results show that surface-attached FBGs has good strain transfer effect, high measurement accuracy and good consistency with traditional strain gauge measurement methods. In addition, we also found that the surface-attached FBGs is greatly affected by noise. Therefore, in addition to strictly operating according to the experimental procedure, we also need to avoid the influence of temperature and noise on the test to improve the test accuracy. This experiment provides a reference for the application of surface-attached FBGs in the strain measurement of aircraft wings, and lays a foundation for the realization of the full-field deformation measurement of aircraft wings. We will further study the application of distributed measurement of surface-attached FBGs in transfer alignment.

\section{Acknowledgements}

This work is supported by the National Natural Science Foundation of China (No. 61873064 and No. 51375087), the National Defense Advanced Research Foundation of China.

\section{References}

1. Qiao, X. G., Zhang, L., Jia, Z. A., GAO, H., \& MA, C. Research on FBG calibration demodulation system based on tunable fiber fabry-perot filter. Transducer and Microsystem Technologies, 27.1, (2008)

2. Zhang, Z., Wang, Y., Sun, Y., Zhang, Q., You, Z., \& Huang, X. Analysis and experimental study on the strain transfer mechanism of an embedded basalt fiber-encapsulated fiber Bragg grating sensor. Optical Engineering, 56.1, (2017)

3. Richter-Trummer, V., Silva, S. O., Peixoto, D. F. C., Frazao, O., Moreira, P. M. G. P., Santos, J. L., \& De Castro, P. M. S. T. Fibre Bragg grating sensors for monitoring the metal inert gas and friction stir welding processes. Measurement Science and Technology 21.8 (2010)

4. Roths, J., Wilfert, A., Kratzer, P., Jülich, F., \& Kuttler, R. Strain calibration of optical FBG-based strain sensors. Fourth European Workshop on Optical Fibre Sensors. International Society for Optics and Photonics, 7653, (2010)

5. Habel, W. R., Schukar, V. G., \& Kusche, N. Calibration facility for quality certification of surface-attached fiber optic and electrical strain sensors. SENSORS, 2011 IEEE. IEEE,( 2011)

6. Lazarev, V. A., Leonov, S. O., Tarabrin, M. K., \& Karasik, V. E. Fiber Bragg gratings strain measuring system and a sensor calibration setup based on mechanical nanomotion transducer. Optical Measurement Systems for Industrial Inspection X. International Society for Optics and Photonics, 10329 (2017). 